\title{
MicroRNA-379-5p plays a tumor-suppressive role in human bladder cancer growth and metastasis by directly targeting MDM2
}

\author{
DEYAO WU ${ }^{1,2^{*}}$, XIAOBING NIU ${ }^{1,3^{*}}$, JUN TAO $^{1 *}$, PENGCHAO LI $^{1}$, QIANG LU ${ }^{1}$, \\ AIMING XU $^{1}$, WEI CHEN ${ }^{1}$ and ZENGJUN WANG ${ }^{1}$ \\ ${ }^{1}$ State Key Laboratory of Reproductive Medicine and Department of Urology, The First Affiliated Hospital of Nanjing \\ Medical University, Nanjing, Jiangsu; ${ }^{2}$ Department of Urology, The Fourth Affiliated Hospital of \\ Nantong Medical College, Yancheng City No. 1 People's Hospital, Yancheng, Jiangsu; \\ ${ }^{3}$ Department of Urology, Huai'an First People's Hospital, Nanjing Medical University, \\ Huai'an, Jiangsu, P.R. China
}

Received October 8, 2016; Accepted November 22, 2016

DOI: $10.3892 /$ or.2017.5607

\begin{abstract}
Bladder cancer is the second most common urological malignancy in the US and is the most frequently diagnosed urological malignancy in China. An increasing amount of evidence indicates that microRNAs perform extremely important functions in many biological processes related to the formation and progression of cancers, including bladder cancer. Previous studies have reported that microRNA-379-5p (miR-379-5p) is involved in tumour initiation and development in human cancers. However, the expression pattern, biological functions and the underlying mechanisms of miR-379-5p in bladder cancer remain unknown. The present study demonstrated that the expression levels of miR-379-5p in bladder cancer tissues and cell lines were lower than the levels in adjacent normal tissues and the human bladder epithelial immortalized SV-HUC-1 cell line. Restoration of the expression of miR-379-5p inhibited bladder cancer cell proliferation, migration and invasion. Mouse double minute 2 (MDM2) was identified as a direct target gene of miR-379-5p. Furthermore, similar to miR-379-5p overexpression in bladder cancer cells, inhibition of MDM2 exerted tumor-suppressive effects. Rescue experiments showed that upregulation of MDM2 reversed the inhibitory effects of miR-379-5p on bladder cancer cell proliferation, migration and invasion. MDM2 was
\end{abstract}

Correspondence to: Professor Zengjun Wang, State Key Laboratory of Reproductive Medicine and Department of Urology, The First Affiliated Hospital of Nanjing Medical University, 300 Guangzhou Road, Nanjing, Jiangsu 210029, P.R. China

E-mail: zengjunwang@njmu.edu.cn

*Contributed equally

Key words: microRNA-379-5p, mouse double minute 2, proliferation, migration, invasion, bladder cancer highly expressed and inversely correlated with miR-379-5p expression in bladder cancer tissues. These findings suggest that the miR-379-5p/MDM2 pathway plays an important role in bladder cancer and could serve as a potential candidate for bladder cancer therapeutics.

\section{Introduction}

Bladder cancer is the second most common urological malignancy in the US and is by far the most frequently diagnosed urological malignancy in China. Epidemiological data provided by the International Agency for Research on Cancer (IARC) in 2012 reported 55,486 cases and 26,820 deaths of bladder cancer in China (1). A total of 740,000 new cases and 16,000 deaths caused by bladder cancer were recorded in 2015 in the US (2). Genetic polymorphisms, chromosomal anomalies and genetic and epigenetic alterations are responsible for the formation and progression of tumors in bladder cancer (3). The most common type of bladder cancer is urothelial transitional cell carcinoma, which accounts for $92-99 \%$ of the total cases diagnosed in North America, Europe and Australia, 70-80\% in Southeast Asia and less than 50\% in different parts of Africa (4). The main therapeutic method for bladder cancer without metastasis is surgery followed by postoperative intravesical instillation (5). Despite the significant advances in surgical techniques and adjuvant chemotherapy, bladder cancer remains a highly prevalent and lethal malignancy (6). Therefore, new sensitive and reliable biomarkers and new therapeutic targets and approaches must be established to treat bladder cancer.

MicroRNAs (miRNAs) are a large group of single-strand, endogenous, non-coding, short RNAs containing 22 nucleotides (7). miRNAs make up a novel class of post-transcriptional gene regulators through interaction with partially complementary target sites at the $3^{\prime}$ untranslated regions (3' UTRs) of their target genes either by inducing their degradation or impairing their translation $(8,9)$. More than 1,000 miRNAs have been identified in the human genome (http://www.mirbase.org/), and 
these miRNAs can regulate $\sim 60 \%$ of all human genes $(10,11)$. miRNAs play a crucial role in cell proliferation, cycle, apoptosis, angiogenesis, tumor progression, metastasis and many other physiological and pathologic processes $(12,13)$. A number of studies have reported that abnormally expressed miRNAs are involved in the initiation and progression of several human diseases, such as atherosclerosis, diabetes, migraine and cancer (14-16). Recently, miRNAs have been identified as tumour suppressors or oncogenes in tumor onset and development (17). Therefore, miRNAs can be utilized to treat malignant conditions.

miR-379-5p has been studied in several types of human cancer (18-20), but information concerning miR-379-5p in bladder cancer is unavailable. In the present study, the expression of miR-379-5p in bladder cancer tissues and cell lines was evaluated. The effects of miR-379-5p transfection on bladder cancer cells were also evaluated in vitro to elucidate the functions and mechanisms of miR-379-5p in bladder cancer initiation and progression. The results of the present study may contribute towards identifying a novel therapeutic target for the treatment of bladder cancer.

\section{Materials and methods}

Clinical specimens. The present study was approved by the Ethics Committee of The First Affiliated Hospital of Nanjing Medical University and performed in accordance with the ethical standards of the Declaration of Helsinki. Informed consent was obtained from all patients prior to the collection of specimens. Twenty-seven paired bladder cancer and adjacent normal tissues were collected from patients who had undergone radical cystectomy at The First Affiliated Hospital of Nanjing Medical University (Nanjing, China) between January 2011 and August 2014. None of the patients were treated with chemotherapy or radiotherapy prior to surgery. Fresh tissues were immediately snap-frozen in liquid nitrogen and stored at $-80^{\circ} \mathrm{C}$.

Cell lines and culture conditions. Human bladder cancer cell lines (T24, EJ and TCCSUP) and the human bladder epithelial immortalized SV-HUC-1 cell line were purchased from Shanghai Institute of Biochemistry and Cell Biology (Shanghai, China). All cells were cultured in Dulbecco's modified Eagle's medium (DMEM) or F12 medium with $10 \%$ fetal bovine serum (FBS), $100 \mathrm{U} / \mathrm{ml}$ penicillin and $100 \mathrm{mg} / \mathrm{ml}$ streptomycin (all from Gibco, Grand Island, NY, USA) in a humidified incubator at $37^{\circ} \mathrm{C}$ with $5 \% \mathrm{CO}_{2}$.

Quantitative reverse-transcription polymerase chain reaction (RT-qPCR). Total RNA was harvested from tissues and cells with TRIzol reagent (Invitrogen, Carlsbad, CA, USA) according to the manufacturer's instructions. Reverse transcription was conducted with the PrimeScript RT reagent kit (Takara, Dalian, China) following the manufacturer's protocol. miR-379-5p expression was quantified with the TaqMan miRNA assay kit (Applied Biosystems, Foster City, CA, USA) with U6 as an internal control. SYBR Premix Ex Taq ${ }^{\mathrm{TM}}$ kits (Takara, Tokyo, Japan) were used to detect MDM2 mRNA expression with $\beta$-action as an internal control. RT-qPCR was performed with the Applied Biosystems ${ }^{\circledR}$ 7900HT Real-Time
PCR system (Thermo Fisher Scientific, Waltham, MA, USA). The relative expression was analyzed by the $2^{-\Delta \Delta C t}$ method (21). The primers used in the present study were as follows: miR-379-5p, 5'-GCGCTGGTAGACTATGGAA-3' and 5'-GTGCAGGGTCCGAGGT-3'; U6, 5'-CTCGCTTCGG CAGCACATATACT-3' and 5'-ACGCTTCACGAATTTGC GTGTC-3'; MDM2, 5'-CAGGCAAATGTGCAATACCAA-3' and 5'-GGTTACAGCACCATCAGTAGGTACAG-3'; $\beta$-action, 5'-GGAGAATGGCCCAGTCCTC-3' and 5'-GGGC ACGAAGGCTCATCAT-3'.

Transfection. miR-379-5p mimics and miRNA negative control (miR-NC) were obtained from GenePharma Co., Ltd., (Shanghai, China). Small interfering RNA (siRNA) for mouse double minute 2 (MDM2) (si-MDM2), negative control siRNA (si-NC), pcDNA3.1-MDM2 and pcDNA3.1 were synthesized by RiboBio (Guangzhou, China). For transfection, cells were seeded in each well of a 6-well plate at a density of $60 \%$ confluence and transfected with miR-379-5p mimics (50 pmol/ml), miR-NC (50 pmol/ml), si-MDM2 (50 pmol/ml), si-NC $(50 \mathrm{pmol} / \mathrm{ml}), \mathrm{pcDNA} 3.1-\mathrm{MDM} 2(2 \mu \mathrm{g})$ or pcDNA3.1 $(2 \mu \mathrm{g})$ using Lipofectamine 2000 (Invitrogen, Grand Island, NY, USA) following the manufacturer's protocol. After transfection at $48 \mathrm{~h}$, transfection efficiency was determined through RT-qPCR.

Cell Counting Kit-8 (CCK-8) assay. Transfected cells were collected, suspended and re-seeded at 3,000/well on 96-well plates. The cells were then incubated at $37^{\circ} \mathrm{C}$ in a humidified incubator with $5 \% \mathrm{CO}_{2}$. Cell proliferation was evaluated 1 , 2, 3 and 4 days after seeding by the CCK- 8 assay (Dojindo, Kumamoto, Japan). Briefly, $10 \mu \mathrm{l}$ of CCK-8 solution was added to each well. After $2 \mathrm{~h}$ of incubation at $37^{\circ} \mathrm{C}$, an ELISA reader (Bio-Rad, Richmond, CA, USA) was used to measure optical density (OD) at the wavelength of $450 \mathrm{~nm}$. Each experiment was performed in triplicate.

Cell migration and invasion assays. Cell migration and invasion assays were performed in Transwell chambers with an 8- $\mu \mathrm{m}$ pore polycarbonate membrane (BD Biosciences, Franklin Lakes, NJ, USA). For the cell migration assay, transfected cells were collected $48 \mathrm{~h}$ post-transfection and suspended in an FBS-free culture medium. A total of $5 \times 10^{4}$ cells were seeded into the upper chambers, whereas the lower chambers were filled with $500 \mu \mathrm{l}$ of DMEM containing 20\% FBS. Cells were incubated at $37^{\circ} \mathrm{C}$ in a humidified incubator with $5 \%$ $\mathrm{CO}_{2}$ for $48 \mathrm{~h}$. The cells that did not migrate through the pores in the membranes were scraped and washed away. The cells that migrated were fixed with $100 \%$ methanol, stained with $0.5 \%$ crystal violet, washed with phosphate-buffered saline and dried in air. The cells were counted with an inverted microscope (magnification of x200; Olympus, Tokyo, Japan). Cell invasion assays were performed in a similar manner, but the cells were allowed to migrate through Transwell chambers coated with Matrigel (BD Biosciences, San Jose, CA, USA).

Bioinformatic analysis and luciferase reporter assay. TargetScan Human 7.0 (http://www.targetscan.org/) and miRanda (http://www. microrna.org/microrna/) were employed to identify the potential target genes of miR-379-5p. 

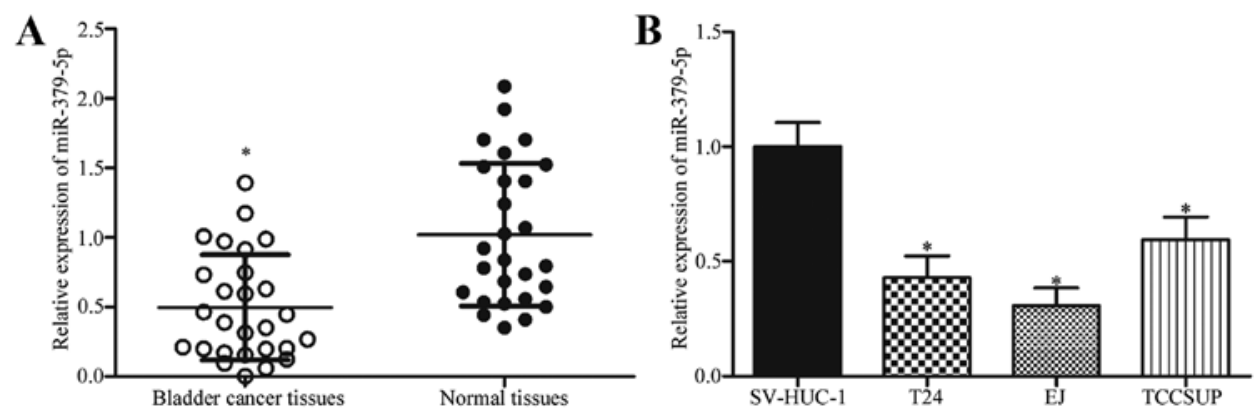

Figure 1. miR-379-5p expression in bladder cancer tissues and cell lines. (A) miR-379-5p was downregulated in bladder cancer tissues. (B) miR-379-5p was underexpressed in bladder cancer cell lines compared with that in the human bladder epithelial immortalized SV-HUC-1 cell line; ${ }^{*} \mathrm{P}<0.05$ compared with the control.

For the luciferase reporter assay, the reporter plasmids were synthesized and purified with GenePharma. The 3' UTR sequence of MDM2, which was predicted to interact with miR-379-5p or a mutated sequence within the predicted target sites, was produced and inserted into the pmirGLO vector. Twenty-four hours before transfection, the cells were seeded on a $24-$ well plate at a density of $30-40 \%$ confluence. Lipofectamine 2000 was used to co-transfect the cells with pmirGLO-MDM2-3' UTR Wt or pmirGLO-MDM2-3' UTR Mut and miR-379-5p mimics or miR-NC. Forty-eight hours after transfection, the cells were harvested for reporter assay. Luciferase activities were detected through dual-luciferase reporter assays (Promega, Manheim, Germany) according to the manufacturer's instructions. Firefly luciferase activities were normalized to Renilla luciferase activities.

Western blotting. Total protein was isolated from tissues and cells with a radioimmunoprecipitation assay lysis buffer [150 mM of $\mathrm{NaCl}, 1 \% \mathrm{NP}-40,0.5 \%$ deoxycholate and $1 \%$ sodium dodecyl sulphate (SDS)] containing protease and phosphatase inhibitors (Thermo Fisher Scientific, Franklin Lakes, MA, USA). A bicinchoninic acid assay kit (Beyotime Institute of Biotechnology, Haimen, China) was used to determine the concentration of total protein. Equal amounts of proteins were separated through $10 \%$ SDS-polyacrylamide gel (SDS-PAGE) electrophoresis and then transferred to polyvinylidine flouride membranes (Millipore, Billerica, MA, USA). Subsequently, the membranes were blocked in Tris-buffered saline with $0.1 \%$ Tween-20 (TBST) containing 5\% skimmed milk and incubated with primary antibodies, namely, mouse anti-human MDM2 antibody (1:1,000 dilution; sc-965) and mouse anti-human monoclonal GADPH antibody $(1: 1,000$ dilution; sc-365062) (both from Santa Cruz Biotechnology, Santa Cruz, CA, USA), at $4^{\circ} \mathrm{C}$ overnight. After being washed in TBST, the membranes were incubated with goat anti-mouse (HRP)-conjugated secondary antibody (1:5,000 dilution, sc-2005; Santa Cruz Biotechnology) at room temperature for $1 \mathrm{~h}$. The protein bands were visualized with ECL substrates (Millipore). Glyceraldehyde 3-phosphate dehydrogenase was used as a loading control.

Statistical analysis. Data are presented as means \pm standard deviation and were compared with the Student's t-test or ANOVA using SPSS version 13.0 software (SPSS, Inc.,
Chicago, IL, USA). A P-value of $<0.05$ was considered statistically significant.

\section{Results}

miR-379-5p expression is downregulated in bladder cancer tissues and cell lines. To determine whether miR-379-5p contributes to the progression of bladder cancer, we determined the miR-379-5p expression in bladder cancer and adjacent normal tissues by RT-qPCR. As shown in Fig. 1A, miR-379-5p in bladder cancer tissues was underexpressed compared with that in adjacent normal tissues $(\mathrm{P}<0.05)$. Subsequently, the expression levels of miR-379-5p in 3 bladder cancer cell lines and human bladder epithelial immortalized SV-HUC-1 cell line were determined. The results showed that miR-379-5p in bladder cancer cell lines was underexpressed compared with that in the SV-HUC-1 cell line (Fig. 1B; P<0.05). These results suggest that miR-379-5p is frequently downregulated in bladder cancer and may play important roles in the progression of bladder cancer.

miR-379-5p suppresses bladder cancer cell proliferation, migration and invasion. To determine the function of miR-379-5p in bladder cancer, miR-379-5p mimics were introduced into T24 and EJ cells to increase the expression level of miR-379-5p. Transfection efficiency was evaluated through RT-qPCR $48 \mathrm{~h}$ post-transfection. As shown in Fig. 2A, miR-379-5p expression was markedly increased in the T24 and EJ cells transfected with the miR-379-5p mimics $(\mathrm{P}<0.05)$. CCK- 8 assay was then performed to evaluate the effect of miR-379-5p on cell proliferation in bladder cancer. The results showed that the upregulation of miR-379-5p inhibited T24 and EJ cell proliferation (Fig. 2B; $\mathrm{P}<0.05$ ). Cell migration and invasion assays were performed to further investigate the function of miR-379-5p in the regulation of cell migration and invasion of bladder cancer cells. As shown in Fig. 2C, ectopic expression of miR-379-5p led to a significant decrease in the migration and invasion capacities of the T24 and EJ cells $(\mathrm{P}<0.05)$. These results suggest that miR-379-5p plays a tumorsuppressive role in bladder cancer growth and metastasis.

MDM2 is a direct target of miR-379-5p in bladder cancer. miRNAs mainly function by negatively regulating their target genes; therefore, we investigated the direct target of miR-379-5p. 
A
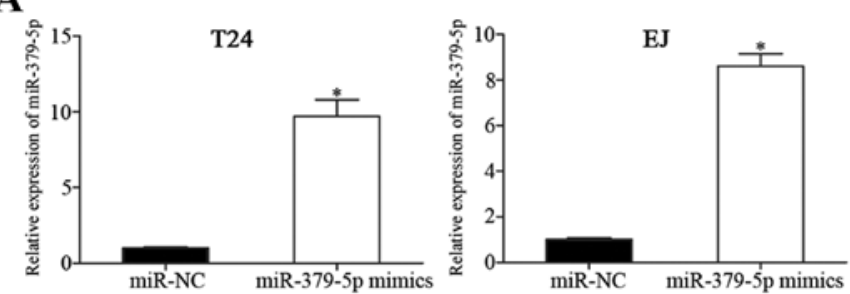

B
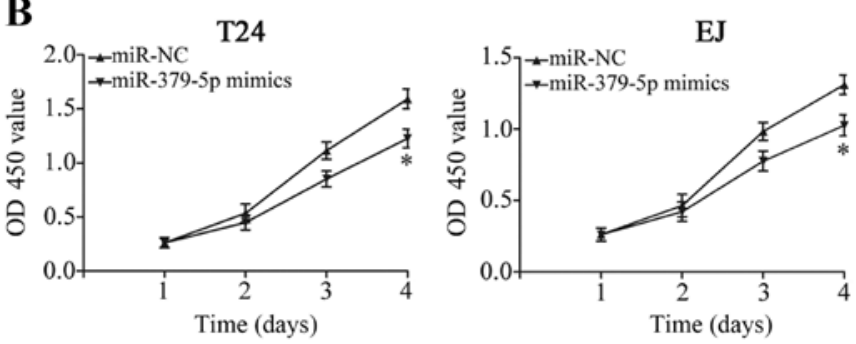

C

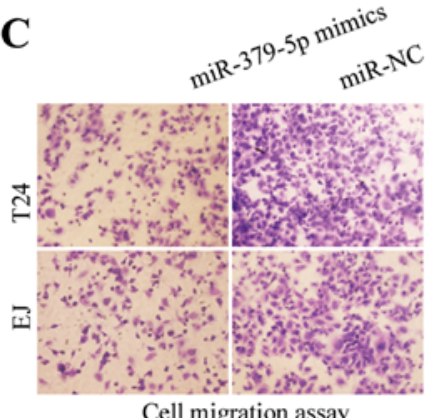

Cell migration assay

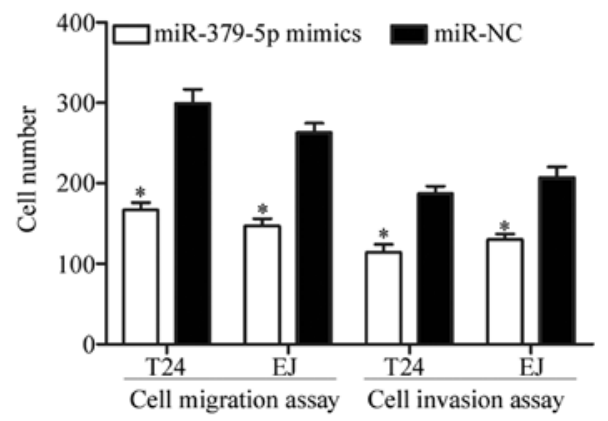

Figure 2. Restoration of miR-379-5p expression inhibits bladder cancer cell proliferation, migration and invasion. (A) RT-qPCR was conducted to assess transfection efficiency in T24 and EJ cells after transfection with miR-379-5p mimics or miR-NC. (B) CCK-8 assay was used to evaluate the proliferation of T24 and EJ cells after transfection with miR-379-5p mimics or miR-NC. (C) Resumption of the expression of miR-379-5p decreased the migration and invasion abilities of the T24 and EJ cells; ${ }^{*} \mathrm{P}<0.05$ compared with the control.

Bioinformatic algorithms (TargetScan and miRanda) were used to predict the potential targets of miR-379-5p. As shown in Fig. 3A, the 3' UTR of MDM2 contains a target sequence for miR-379-5p. To confirm whether MDM2 is a direct target of miR-379-5p, luciferase reporter assay was conducted on T24 and EJ cells. Luciferase activities were evidently decreased in the T24 and EJ cells co-transfected with the miR-379-5p mimics and pmirGLO-MDM2-3' UTR Wt (Fig. 3B; P<0.05). However, the luciferase activities of pmirGLO-MDM2-3' UTR Mut were unaffected.

To further confirm this hypothesis, RT-qPCR and western blotting were employed to evaluate the regulatory effects of
A

$\begin{array}{lrr}\text { MDM2-3' UTR Wt } & 5, & \text {...GUUUGUGAUCAUAUUGUCUACCA... } \\ \text { hsa-miR-379-5p } & 3, & \text { GGAGUCAAGGUAUCAGAUGGU } \\ \text { MDM2-3' UTR Mut } & 5, & \text {...GUUUGUGAUCAUAUUCAGAUGGA... }\end{array}$

B
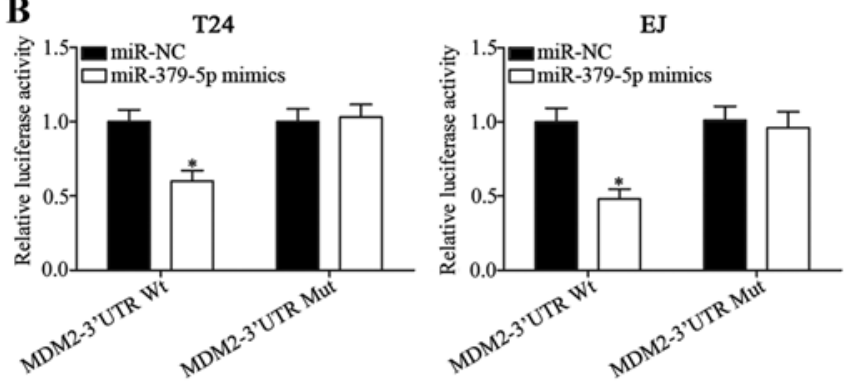

C

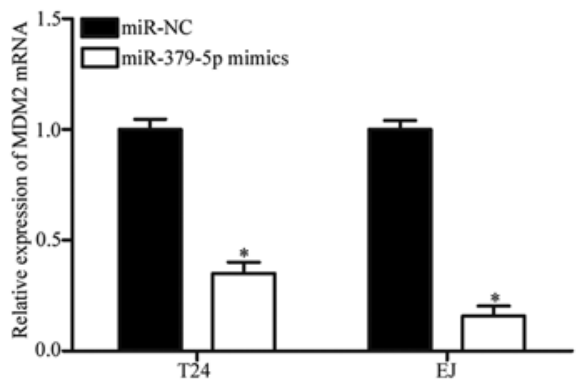

D

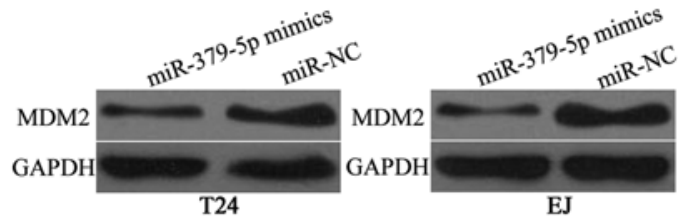

Figure 3. miR-379-5p directly targets MDM2 in bladder cancer. (A) Schematic of miR-379-5p putative binding sites in the 3' UTR of MDM2 and its mutated version. (B) Relative luciferase activities were determined in T24 and EJ cells co-transfected with pmirGLO-MDM2-3' UTR Wt or pmirGLOMDM2-3' UTR Mut and miR-379-5p mimics or miR-NC. MDM2 expression at the (C) mRNA and (D) protein level in T24 and EJ cells transfected with miR-379-5p mimics or miR-NC was determined through RT-qPCR and western blotting; ${ }^{\mathrm{P}}<0.05$ compared with the control.

miR-379-5p on endogenous MDM2 expression in the T24 and EJ cells. As shown in Fig. 3C and D, MDM2 mRNA and protein expression were significantly reduced in the T24 and EJ cells transfected with miR-379-5p mimics compared with cells transfected with miR-NC (both $\mathrm{P}<0.05$ ). Collectively, these findings suggest that miR-379-5p negatively regulates MDM2 expression by directly binding to the 3' UTR of its mRNA in bladder cancer.

Inhibition of MDM2 exerts similar effects as miR-379-5p overexpression in bladder cancer. To determine the involvement of MDM2 in bladder cancer, the biological role of MDM2 in cell proliferation, migration and invasion were examined. As shown in Fig. 4A, MDM2 protein was underexpressed in the T24 and EJ cells after transfection with si-MDM2 (P<0.05). CCK-8 assay and cell migration and invasion assays showed that inhibition of MDM2 expression suppressed the growth (Fig. 4B; $\mathrm{P}<0.05$ ) and metastasis (Fig. 4C; $\mathrm{P}<0.05$ ) of T24 and EJ cells. These results suggest that MDM2 knockdown exerts a suppressive effect similar to miR-379-5p overexpression in bladder cancer and further confirm that MDM2 is a functional target of miR-379-5p. 

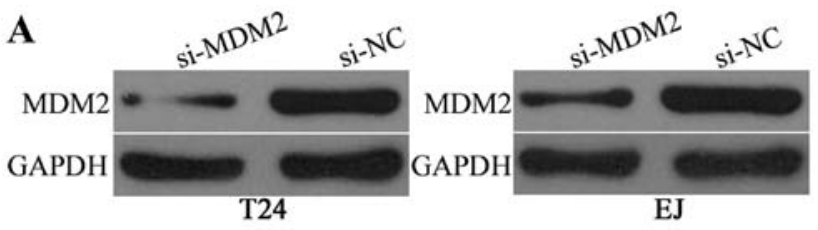

B
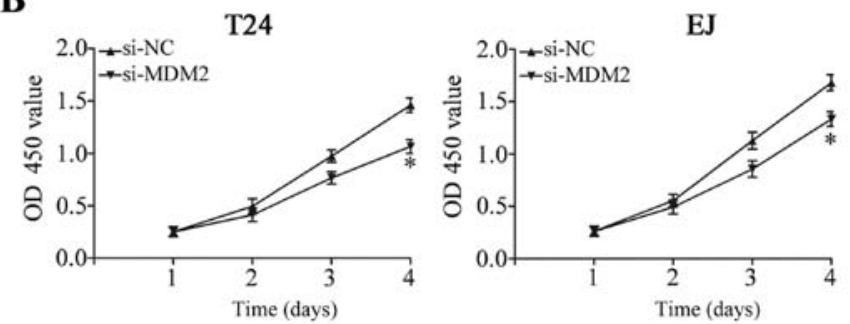

C

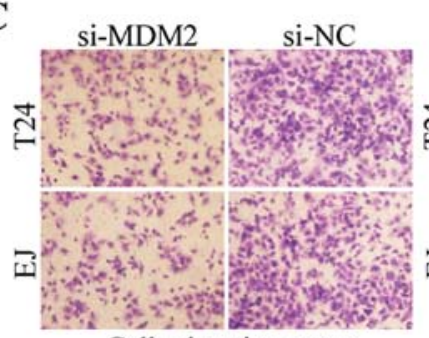

Cell migration assay

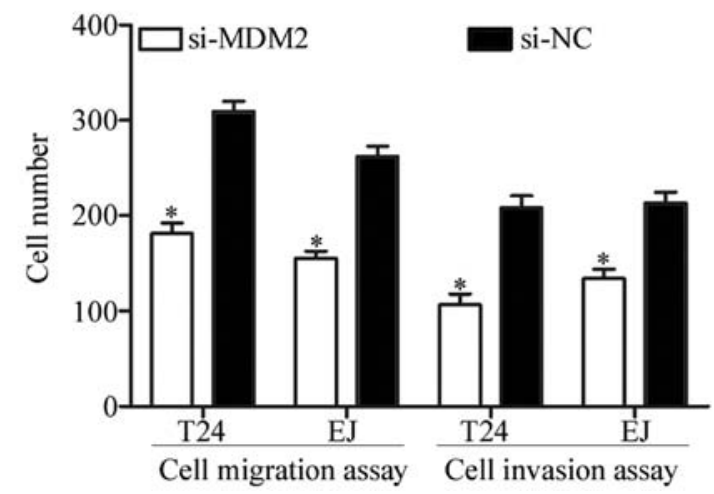

Figure 4. Effects of MDM2 knockdown on the biological behaviors of T24 and EJ cells. (A) Western blot analysis of MDM2 protein expression in the T24 and EJ cells transfected with si-MDM2 or si-NC. (B) CCK-8 assay showed that MDM2 knockdown suppressed T24 and EJ cell proliferation in vitro. (C) Downregulation of MDM2 reduced the migration and invasion abilities of T24 and EJ cells; ${ }^{*} \mathrm{P}<0.05$ compared with the control.

Enforced expression of MDM2 reverses miR-379-5p-induced effects on cell proliferation, migration and invasion in bladder cancer. A 'rescue' experiment was performed to determine whether miR-379-5p inhibits the growth and metastasis of bladder cancer cells by targeting MDM2. pcDNA3.1-MDM2 was transfected into T24 and EJ cells to increase its expression (Fig. 5A; $\mathrm{P}<0.05)$. The expression level of MDM2 protein in the T24 and EJ cells was recovered after co-treatment with miR-379-5p mimics and pcDNA3.1-MDM2 (Fig. 5B; $\mathrm{P}<0.05$ ). Moreover, resumption of expression of MDM2 restored proliferation (Fig. 5C; $\mathrm{P}<0.05$ ), migration and invasion (Fig. 5D; $\mathrm{P}<0.05$ ) induced by miR-379-5p overexpression in the T24 and EJ cells. These results suggest that miR-379-5p suppresses bladder cancer cell proliferation, migration and invasion partly by targeting MDM2.

miR-379-5p is negatively correlated with MDM2 expression in bladder cancer tissues. We analyzed the association between
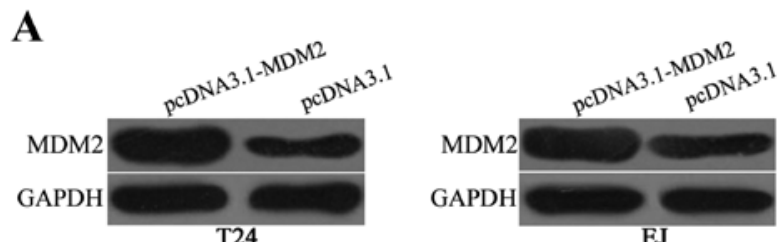

B

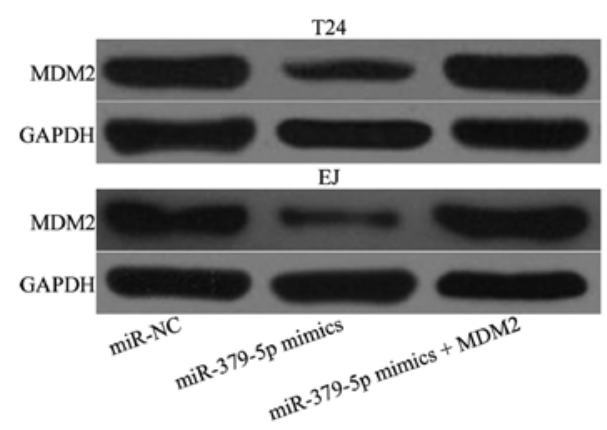

C
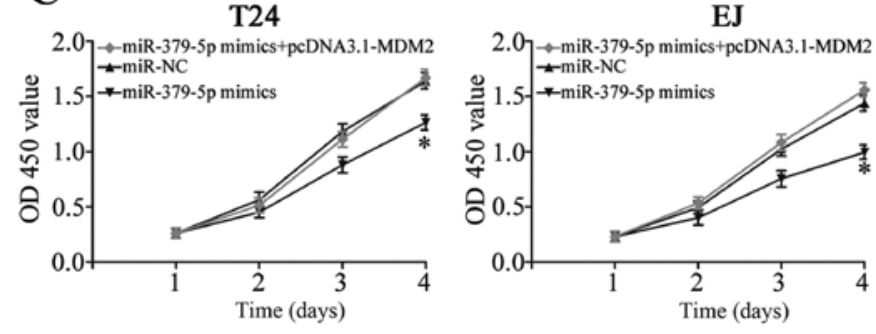

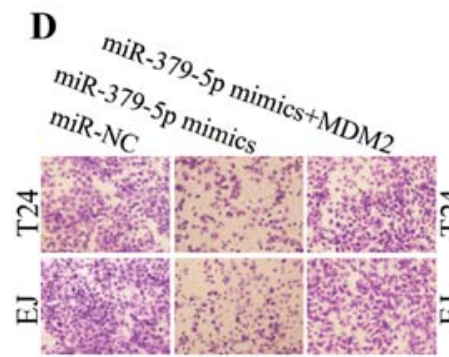

Cell migration assay

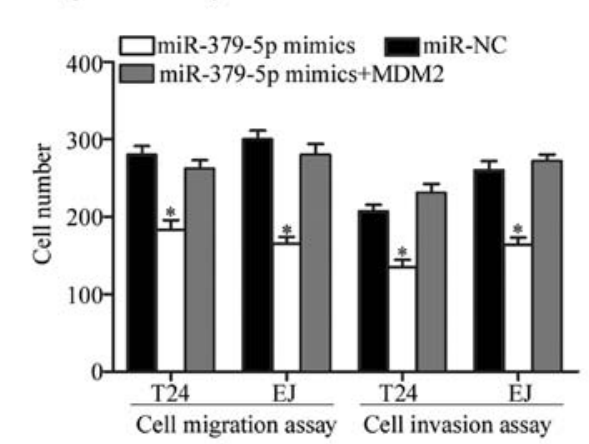

Figure 5. MDM2 is an important functional mediator of miR-379-5p in T24 and EJ cells. (A) Western blot results showed that MDM2 protein was upregulated in pcDNA3.1-MDM2-transfected T24 and EJ cells. (B) Western blot results showed that MDM2 protein in T24 and EJ cells was recovered after co-treatment with miR-379-5p mimics and pcDNA3.1-MDM2. (C) CCK-8 assay showed that the ectopic expression of MDM2 rescued the proliferation induced by miR-379-5p overexpression in T24 and EJ cells. (D) Cell migration and invasion assays revealed that the exogenous expression of MDM2 rescued the migration and invasion abilities induced by miR-379-5p overexpression in T24 and EJ cells; " $\mathrm{P}<0.05$ compared with the control.

the expression levels of miR-379-5p and MDM2 in clinical bladder cancer tissues. MDM2 mRNA was higher in the 
A

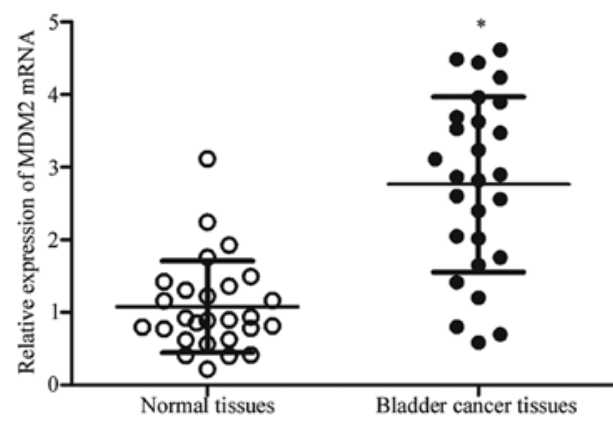

B

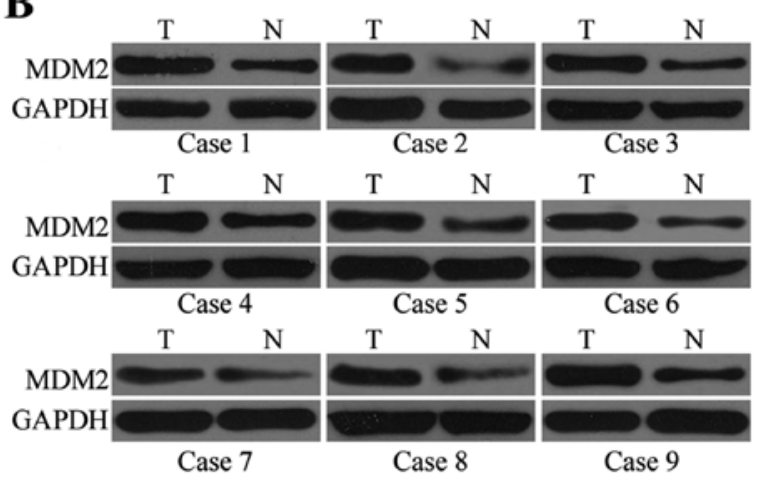

C

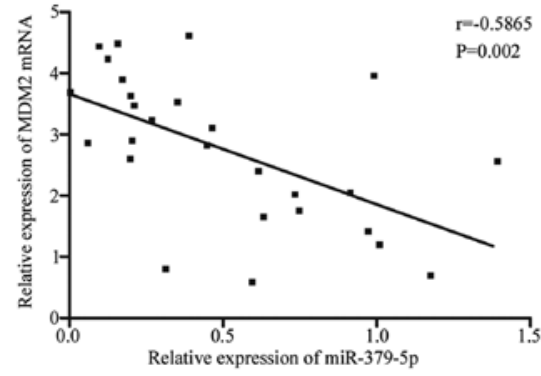

Figure 6. MDM2 is upregulated in bladder cancer tissues and inversely correlated with miR-379-5p expression. (A) RT-qPCR was conducted to determine MDM2 mRNA expression in bladder cancer tissues and their matching adjacent normal tissues. (B) Western blotting was performed to examine MDM2 protein expression in bladder cancer tissues and their matching adjacent normal tissues. (C) MDM2 mRNA expression in bladder cancer tissues was negatively correlated with miR-379-5p expression; ${ }^{\mathrm{P}} \mathrm{P}<0.05$ compared with the control.

bladder cancer tissues than that noted in the adjacent normal tissues (Fig. 6A; P<0.05). Western blot results indicated that MDM2 protein expression in the bladder cancer tissues was upregulated compared with that in the adjacent normal tissues (Fig. 6B; $\mathrm{P}<0.05$ ). Moreover, Spearman's correlation analysis indicated an inverse correlation between miR-379-5p and MDM2 mRNA expression in the bladder cancer tissues (Fig. 6C; $r=-0.5865 ; \mathrm{P}=0.002$ ). These results further confirm that MDM2 is a direct target gene of miR-379-5p in bladder cancer.

\section{Discussion}

An increasing amount of evidence indicates that miRNAs play important roles in many biological processes related to carcinogenesis and progression, such as cell proliferation, apoptosis, cell cycle, invasion and metastasis (22-24). Furthermore,
miRNAs represent novel diagnostic and therapeutic targets for the treatment of human cancer (25). In the present study, miR-379-5p was downregulated in bladder cancer tissues and cell lines. Restoration of the expression of miR-379-5p inhibited bladder cancer cell proliferation, migration and invasion. Moreover, MDM2 was identified as the direct target gene of miR-379-5p. These results suggest that miR-379-5p acts as a tumor suppressor in bladder cancer and may be investigated as an efficient therapeutic target for clinical application. To the best of our knowledge, the present study is the first to investigate the expression patterns, biological functions and underlying mechanism of miR-379-5p in bladder cancer.

An increasing number of studies have shown that miR-379-5p, which is located on chromosome 14q32.31, plays important roles in tumorigenesis and tumor development. For instance, Khan et al reported that miR-379-5p is downregulated in breast cancer tissues compared with that in normal breast tissues. The low expression level of miR-379-5p was found to be significantly correlated with tumor stage. miR-379-5p re-expression suppressed cell proliferation by negatively regulating cyclin B1 (18). Chen et al found that miR-379-5p expression is lower in hepatocellular carcinoma tissues and cell lines. Reduced miR-379-5p was found to be associated with the TNM stage and metastasis of patients. Restoration of the expression of miR-379-5p inhibited cell migration, invasion, epithelial-to-mesenchymal transition (EMT) and metastasis both in vitro and in vivo by directly targeting focal adhesion kinase (FAK), thus leading to the suppression of AKT signaling (19). Chen et al revealed that upregulation of miR-379-5p decreased cell migration and invasion by downregulation of MMP-2 and MMP-9 (20). Furthermore, miR-379-5p was highly expressed in bone metastatic prostate cancer tissues and cell lines and was significantly correlated with progression-free survival of patients with prostate cancer. Downregulation of miR-379-5p reduced prostate cancer cell EMT and invasive ability (26). These findings suggest that miR-379-5p can be a diagnostic and prognostic biomarker for human cancers.

Identification of the targets of miRNAs is critical for understanding its role in tumorigenesis (27). To investigate the mechanisms underlying the suppression of bladder cancer cell proliferation, migration and invasion induced by miR-379-5p, we performed bioinformatic analysis and found that the 3' UTR of MDM2 contains a complementary site for the seed region of miR-379-5p. Through a luciferase reporter assay, we demonstrated that miR-379-5p directly targeted the 3' UTR of MDM2. In addition, miR-379-5p overexpression decreased the expression of endogenous MDM2 both at the mRNA and protein levels in bladder cancer cells. Our experimental data further showed that inhibition of MDM2 exerted a suppressive effect similar to miR-379-5p overexpression in bladder cancer cells. MDM2 overexpression rescued miR-379-5p-induced effects on bladder cancer cell proliferation, migration and invasion. Moreover, MDM2 was highly expressed in bladder cancer tissues compared with that in adjacent normal tissues, which was negatively correlated with miR-379-5p expression patterns. These results suggest that miR-379-5p plays a tumor-suppression role in bladder cancer partly by targeting MDM2.

MDM2, which is located on chromosome 12q13.14, is a proto-oncogene that was firstly identified in a locus amplified 
on double minute chromosomes in a tumorigenic mouse cell line (3T3-DM) (28). Numerous studies have reported that the MDM2 gene is amplified or overexpressed in numerous types of human cancers, such as breast (29), lung (30) and colorectal cancer (31), and testicular germ cell tumors (32). In bladder cancer, a study conducted by Lianes et al showed that MDM2 was upregulated in tumor tissues and displayed a significant correlation with low-stage, low-grade bladder tumors of bladder cancer patients (33). Tuna et al revealed that MDM2 overexpression exhibits a significant association with tumor grade and recurrence of bladder cancer and may be a valuable parameter in predicting recurrence (34). Studies have also shown that MDM2 plays important roles during the tumorigenesis and progression of bladder cancer $(35,36)$. Consistent with previous findings, our results demonstrated that MDM2 knockdown exerts anti-proliferative and anti-metastasis effects on bladder cancer. MDM2 is a potential prognostic and therapeutic target for patients with bladder cancer.

In conclusion, miR-379-5p was downregulated in bladder cancer tissues and cell lines and suppressed cell proliferation, migration and invasion by directly targeting MDM2. These results suggest that $\mathrm{miR}-379-5 \mathrm{p} / \mathrm{MDM} 2$-based targeted therapy may be a promising therapeutic treatment for bladder cancer in the future.

\section{Acknowledgements}

The present study was supported by a grant from the National Natural Science Foundation of China (no. 81270685).

\section{References}

1. Zhang Y, Sun Y, Chen T, Hu H, Xie W, Qiao Z, Ding N, Xie L, Li S, Wang W, et al: Genetic variations rs11892031 and rs401681 are associated with bladder cancer risk in a Chinese population. Int J Mol Sci 15: 19330-19341, 2014.

2. Siegel RL, Miller KD and Jemal A: Cancer statistics, 2015. CA Cancer J Clin 65: 5-29, 2015.

3. Knowles MA: Molecular pathogenesis of bladder cancer. Int J Clin Oncol 13: 287-297, 2008.

4. Scélo $\mathrm{G}$ and Brennan P: The epidemiology of bladder and kidney cancer. Nat Clin Pract Urol 4: 205-217, 2007.

5. Wu D, Zhou Y, Pan H, Zhou J, Fan Y and Qu P: microRNA-99a inhibiting cell proliferation, migration and invasion by targeting fibroblast growth factor receptor 3 in bladder cancer. Oncol Lett 7: 1219-1224, 2014.

6. Kim WJ and Bae SC: Molecular biomarkers in urothelial bladder cancer. Cancer Sci 99: 646-652, 2008.

7. Ambros V: MicroRNA pathways in flies and worms: Growth, death, fat, stress, and timing. Cell 113: 673-676, 2003.

8. Bartel DP: MicroRNAs: Target recognition and regulatory functions. Cell 136: 215-233, 2009.

9. Bartel DP: MicroRNAs: Genomics, biogenesis, mechanism, and function. Cell 116: 281-297, 2004.

10. Wu W, Sun M, Zou GM and Chen J: MicroRNA and cancer: Current status and prospective. Int J Cancer 120: 953-960, 2007.

11. Nelson KM and Weiss GJ: MicroRNAs and cancer: Past, present, and potential future. Mol Cancer Ther 7: 3655-3660, 2008.

12. Garzon R, Fabbri M, Cimmino A, Calin GA and Croce CM: MicroRNA expression and function in cancer. Trends Mol Med 12: 580-587, 2006.

13. Alvarez-Garcia I and Miska EA: MicroRNA functions in animal development and human disease. Development 132: 4653-4662, 2005.

14. Tana C, Giamberardino MA and Cipollone F: microRNA profiling in atherosclerosis, diabetes and migraine. Ann Med 49: 93-105, 2017.

15. Piletič K and Kunej T: MicroRNA epigenetic signatures in human disease. Arch Toxicol 90: 2405-2419, 2016.
16. Shang Y, Zang A, Li J, Jia Y, Li X, Zhang L, Huo R, Yang J, Feng J, Ge K, et al: MicroRNA-383 is a tumor suppressor and potential prognostic biomarker in human non-small cell lung caner. Biomed Pharmacother 83: 1175-1181, 2016.

17. Calin GA and Croce CM: MicroRNA signatures in human cancers. Nat Rev Cancer 6: 857-866, 2006.

18. Khan S, Brougham CL, Ryan J, Sahrudin A, O'Neill G, Wall D, Curran C, Newell J, Kerin MJ and Dwyer RM: miR-379 regulates cyclin B1 expression and is decreased in breast cancer. PLoS One 8: e68753, 2013.

19. Chen JS, Li HS, Huang JQ, Dong SH, Huang ZJ, Yi W, Zhan GF, Feng JT, Sun JC and Huang XH: MicroRNA-379-5p inhibits tumor invasion and metastasis by targeting FAK/AKT signaling in hepatocellular carcinoma. Cancer Lett 375: 73-83, 2016.

20. Chen JS, Huang JQ, Dong SH and Huang XH: Effects of microRNA-379-5p on proliferation, migration and invasion of hepatocellular carcinoma cell line. Zhonghua Yi Xue Za Zhi 96: 1450-1453, 2016 (In Chinese).

21. Livak KJ and Schmittgen TD: Analysis of relative gene expression data using real-time quantitative PCR and the $2^{-\triangle \Delta C \mathrm{~T}}$ method. Methods 25: 402-408, 2001.

22. Zhong XY, Yu JH, Zhang WG, Wang ZD, Dong Q, Tai S, Cui YF and Li H: MicroRNA-421 functions as an oncogenic miRNA in biliary tract cancer through down-regulating farnesoid $\mathrm{X}$ receptor expression. Gene 493: 44-51, 2012.

23. Wu D, Niu X, Pan H, Zhou Y, Qu P and Zhou J: MicroRNA-335 is downregulated in bladder cancer and inhibits cell growth, migration and invasion via targeting ROCK1. Mol Med Rep 13: 4379-4385, 2016

24. Wang X, Liu Y, Liu X, Yang J, Teng G, Zhang L and Zhou C: MiR-124 inhibits cell proliferation, migration and invasion by directly targeting SOX9 in lung adenocarcinoma. Oncol Rep 35: 3115-3121, 2016.

25. Baker M: RNA interference: MicroRNAs as biomarkers. Nature 464: 1227, 2010.

26. Gururajan M, Josson S, Chu GC, Lu CL, Lu YT, Haga CL, Zhau HE, Liu C, Lichterman J, Duan P, et al: miR-154* and miR-379 in the DLK1-DIO3 microRNA mega-cluster regulate epithelial to mesenchymal transition and bone metastasis of prostate cancer. Clin Cancer Res 20: 6559-6569, 2014.

27. Osada $\mathrm{H}$ and Takahashi T: MicroRNAs in biological processes and carcinogenesis. Carcinogenesis 28: 2-12, 2007.

28. Pichiorri F, Suh SS, Rocci A, De Luca L, Taccioli C, Santhanam R, Zhou W, Benson DM Jr, Hofmainster C, Alder H, et al: Downregulation of p53-inducible microRNAs 192, 194, and 215 impairs the p53/MDM2 autoregulatory loop in multiple myeloma development. Cancer Cell 18: 349-351, 2016.

29. Marchetti A, Buttitta F, Girlando S, Dalla Palma P, Pellegrini S, Fina P, Doglioni C, Bevilacqua $\mathrm{G}$ and Barbareschi M: $m d m 2$ gene alterations and $\mathrm{mdm} 2$ protein expression in breast carcinomas. $\mathrm{J}$ Pathol 175: 31-38, 1995.

30. Marchetti A, Buttitta F, Pellegrini S, Merlo G, Chella A, Angeletti CA and Bevilacqua G: mdm2 gene amplification and overexpression in non-small cell lung carcinomas with accumulation of the p53 protein in the absence of p53 gene mutations. Diagn Mol Pathol 4: 93-97, 1995.

31. Zhang C, Liu J, Wang X, Wu R, Lin M, Laddha SV, Yang Q, Chan CS and Feng Z: MicroRNA-339-5p inhibits colorectal tumorigenesis through regulation of the MDM2/p53 signaling. Oncotarget 5: 9106-9117, 2014.

32. Riou G, Barrois M, Prost S, Terrier MJ, Theodore C and Levine AJ: The $p 53$ and $m d m-2$ genes in human testicular germ-cell tumors. Mol Carcinog 12: 124-131, 1995.

33. Lianes P, Orlow I, Zhang ZF, Oliva MR, Sarkis AS, Reuter VE and Cordon-Cardo C: Altered patterns of MDM2 and TP53 expression in human bladder cancer. J Natl Cancer Inst 86: 1325-1330, 1994.

34. Tuna B, Yörükoğlu K, Tüzel E, Güray M, Mungan U and Kirkali Z: Expression of p53 and $\mathrm{mdm} 2$ and their significance in recurrence of superficial bladder cancer. Pathol Res Pract 199: 323-328, 2003.

35. Schmitz-Dräger BJ, Kushima M, Goebell P, Jax TW, Gerharz CD, Bültel H, Schulz WA, Ebert T and Ackermann R: p53 and MDM2 in the development and progression of bladder cancer. Eur Urol 32: 487-493, 1997.

36. Shiina H, Igawa M, Shigeno K, Yamasaki Y, Urakami S, Yoneda T, Wada Y, Honda S and Nagasaki M: Clinical significance of $\mathrm{mdm} 2$ and $\mathrm{p} 53$ expression in bladder cancer. A comparison with cell proliferation and apoptosis. Oncology 56: 239-247, 1999. 